\title{
Seed Storability of CIMMYT Core Wheat Germplasm Panel and Their Haplotypes in Lipoxygenase Locus
}

\author{
Yumin Yang1,2*, Jipeng $\mathbf{Q u}^{3^{*}}$, Jing Zhang4, Shijie Xiang5, Jun Li1,6\#, Hongshen Wan ${ }^{1,6 \#}$ \\ ${ }^{1}$ Key Laboratory of Wheat Biology and Genetic Improvement on Southwestern China (Ministry of Agriculture and Rural Areas), \\ Chengdu, China \\ ${ }^{2}$ Institute of Agricultural Resources and Environment, Sichuan Academy of Agricultural Sciences, Chengdu, China \\ ${ }^{3}$ Panxi Crops Research and Utilization Key Laboratory of Sichuan Province, Xichang University, Xichang, China \\ ${ }^{4}$ Food Safety Detection Key Laboratory of Sichuan, Technology Center of Chengdu Customs, Chengdu, China \\ ${ }^{5}$ Sichuan Agricultural products Quality and Safety Center, Chengdu, China \\ ${ }^{6}$ Crop Research Institute, Sichuan Academy of Agricultural Sciences, Chengdu, China \\ Email: "wanhongshen@126.com, ${ }^{*}$ lijunchd@126.com
}

How to cite this paper: Yang, Y.M., Qu, J.P., Zhang, J., Xiang, S.J., Li, J. and Wan, H.S. (2021) Seed Storability of CIMMYT Core Wheat Germplasm Panel and Their Haplotypes in Lipoxygenase Locus. Agricultural Sciences, 12, 977-989. https://doi.org/10.4236/as.2021.129063

Received: August 3, 2021

Accepted: September 12, 2021

Published: September 15, 2021

Copyright $\odot 2021$ by author(s) and Scientific Research Publishing Inc. This work is licensed under the Creative Commons Attribution International License (CC BY 4.0).

http://creativecommons.org/licenses/by/4.0/

\begin{abstract}
Seed storability (SS), also called seed longevity, is a valuable trait for seed banks and providing reliable crop seeds to farmers, which is usually negatively correlated to lipoxygenase (LOX) activity. In this study, the seed storability of 60 accessions of CIMMYT core wheat germplasm panel (CIMCOG) was investigated through artificial aging (AA) test, including three parameters relative germination potential (RGP), relative germination rate (RGR) and relative seedling vigor index (RVI). Significant positive relationships were observed among RGP, RGR and RVI. And the genotypes at three LOX activity related QTLs/genes $Q L p x$.caas- $4 B, Q L p x$.caas- $1 A L$ and TaLOX-B1 were also identified with published trait-associated molecular markers. For $Q L p x . c a a s-4 B$, a total of five alleles were detected at the locus of Xgwm251, and one marker-trait association was identified for RVI. Four and two alleles were detected at the loci of $Q L p x$.caas- $1 A L$ and TaLoxB1 that were significantly associated with RGP, RGR and RVI, respectively. A total of 9 haplotypes were detected at three lipoxygenase activity related gene loci, and the haplotype of three lipoxygenase loci showed a significant association with RGP, RGR and RVI. The haplotype of Xgwm251-125bp $+X w m c 312_{-247 b p}+$ TaLoX-B1b produced seeds with the best storability in the CIMCOG, which could benefit the breeding for wheat with good seed storability.
\end{abstract}

*Yumin Yang and Jipeng Qu contributed equally to this article.

${ }^{*}$ Corresponding author. 


\section{Keywords}

Wheat, Seed Storability, Lipoxygenase, Trait-Marker Association, Haplotype

\section{Introduction}

Seed storability (SS) or seed longevity is defined as seed viability after dry storage. It's a valuable trait for seed banks and providing reliable crop seeds to farmers. Seed storability is often negatively correlated with the advancement of germination [1]. It is a complex trait influenced by two most important environmental factors: 1) relative humidity, which is related to seed moisture content; 2) temperature, which affects the rate of biochemical processes in seeds [2] [3]. More importantly, seed storability is usually controlled by several genes [4]. Genetic dissection of seed storability has been reported in rice [5] [6] [7], soybean [8] and oilseed rape [9]. Seed storability shows their diversification in different varieties of wheat [10] [11], and genetic studies for storability such as seed longevity [12].

Lipoxygenase activity exerts significant effects on seed storability negatively, and a reduction of lipoxygenase activity is therefore of interest for seed longevity in many crops [13] [14] [15] [16]. In cultivated bread wheat, seeds often obtained much higher lipoxygenase activity [17], owing to the artificial selection for higher flour whiteness for degradation of carotenoids by lipoxygenase [18] [19], while carotenoids can scavenge singlet molecular oxygen and peroxyl radicals to protect plants against oxidative processes, and increase the seed longevity [16] [20].

Lipoxygenase activity of wheat seed is mostly controlled by lipoxygenase (LOX) genes on wheat group 4 and 5 chromosomes [21]. And Hessler et al. [22] and Geng et al. [23] detected a major QTL with the highest LOD score related to lipoxygenase activity around $\mathrm{Xgwm} 251$ on $4 \mathrm{BS}$ in different hexaploid germplasms. Carrera et al. [24] identified a deletion at $L p X-B 1$ locus on $4 \mathrm{BS}$ weakening the lipoxygenase activity of durum wheat significantly. Therefore, this locus decided most of the lipoxygenase activity in the cultivated wheat, and two functional markers LOX16 and LOX18 were developed to identify TaLOX-B1a and Ta$L O X-B 1 b$, corresponding to high and low LOX activity, respectively [23]. In the chromosome arm $1 \mathrm{AL}$, another major QTL was also detected, explaining more than $25 \%$ of the total phenotypic variation for LOX activity, and the linked marker was Xwmc312 [25]. And both SSR sites Xgwm251 and Xwmc312 were significantly associated with lipoxygenase activity in the germplasm pool collected from four major wheat-growing regions of China [25].

In this study, both seed storability and the genotypes of LOX activity related loci were evaluated using AA-test and identified using molecular markers in CIMCOG, respectively. And the relationship between LOX activity related loci and seed storability was also executed using general linear model (GLM). The 
aim of this study is to scan the CIMCOG to find the favor haplotype at three lipoxygenase activity related loci with good seed storability for marker-assisted selection (MAS).

\section{Materials and Methods}

\subsection{Plant Materials}

A total of 60 elite accessions from the Core Germplasm set (CIMCOG) provided by the Mexico International Maize and Wheat Improvement Center were planted at Yangma of Sichuan Province in the growing season of 2012-2013. Each accession was harvested in its maturity stage, the seeds of each accession were air-dried in the shade after threshing by hand, and stored in an airtight container after aluminium phosphide (AlP) treatment for protecting grains from insect pests. The pedigrees of the 60 accessions were shown in Zhang et al. [11].

\subsection{Seed Storability (SS) Measurement}

\subsubsection{Artificial Aging (AA) Test}

AA test was conducted to simulate long-term storage artificially and hence allow for the evaluation of SS. According to Zhang et al. [11], 50 uniform seeds were selected for AA tests with 4 replicates for each accession, and these seeds were dispersedly placed in a stainless metal cage sealed inside a required container adding $2 \mathrm{~cm}$ of deionized and sterilized water to its bottom. The container was placed in an accelerated aging chambers (LH-80, Zhejiang Top Cloud-Agri Technology CO., LTD) and held at $43^{\circ} \mathrm{C} \pm 0.5^{\circ} \mathrm{C} \& 82 \%$ relative humidity for 72 $\mathrm{h}$, and then these treated seeds were air-dried and stored at $4^{\circ} \mathrm{C}$. The blank control without AA treatment was set with 3 replicates.

\subsubsection{Germination and Seedling Vigor Test}

Four replicates of 50 seeds each for AA tests with three replicates for control were subjected to a standard ISTA germination test [26]. The seed germination potential (GP) and rate (GR) were recorded on the $4^{\text {th }}$ and $8^{\text {th }}$ day, respectively. Apart from the absolute germination (in both non-treated and treated seed lots), a relative germination potential (RGP) and rate (RGR) was determined by dividing the rates obtained after AA treatment by that of the non-treated control.

For the seedling vigor, 10 seedlings for each 50 -seed plot were randomly selected from the geminated seedlings without any injury or defect in the $8^{\text {th }}$ day simultaneously, to measure average seedling length (AL). The seedling vigor index (VI) and relative seedling vigor index (RVI) were calculated following the formula described as Abdul-Baki and Anderson [27]: VI = GR $\times$ AL, RVI (\%) = [(VI of AA treatment)/(VI of control) $] \times 100 \%$.

\subsection{Genotyping of Lox Locus}

Genomic DNA was isolated from 2-week-old wheat leaves of each accession by a modified cetyl trimethyl ammonium bromide (CTAB) plant DNA extraction 
method [28]. Young leaves were ground to a powder in liquid nitrogen and transferred to $1.5 \mathrm{ml}$ centrifuge tubes. $600 \mathrm{ul}$ hot CTAB buffer $(2 \%$ CTAB w/v, $20 \mathrm{mM}$ EDTA, 1.4 M NaCl, 1\% PVP, $100 \mathrm{mM}$ Tris, pH8.0) was added to the centrifuge tubes. The mixture was placed at $65^{\circ} \mathrm{C}$ for $5 \mathrm{~min}$; then $600 \mathrm{ul}$ chloroform/isoamyl alcohol (24:1) added and mixed. After centrifugation (12,000 rpm, $10 \mathrm{~min}$ ), DNA was precipitated from the supernatant with 0.6 volumes of isopropanol after 12 hours at $-20^{\circ} \mathrm{C}$. Following centrifugation, the DNA pellet was dried and resuspended in deionized water. The DNA was used to analyze molecular allelic variations of lipoxygenase activity related loci (Lox). The PCR primers used for molecular analysis are shown in Table 1. The PCR amplification programs for these molecular markers and the isolation of amplification DNA fragments were referred to Wan et al. [29].

\subsection{Statistical Analysis}

Pearson correlation analysis for phenotypes, Duncan's multiple range test (DMRT) were made in IBM SPSS Statistics Version 22 package (IBM Corp., Chicago, IL). Population distribution of phenotypic data was performed in MS Excel 2003 and SPSS. Associations between molecular markers and phenotypes were tested by general linear model (GLM) in SPSS statistical package.

\section{Results}

\subsection{Seed Storability Related Traits}

A total of three indexes RGP, RGR and RVI were calculated for the measurement of SS after artificial aging. The frequency distribution, mean, median, phenotypic standard deviation (S.D.) and coefficient of variance (C.V.) of three SS related traits in CIMCOG set are showed in Figure 1. The medians of RGP, RGR, and RVI are more than their means in the population. The C.V. of RVI were more than the other two traits, for that RVI, for that the index of RVI is relevant to both seed germination and seedling growth.

The correlation coefficient among all investigated traits were showed in Table 2.

Table 1. Molecular markers used for detecting the allelic variations of Lox loci.

\begin{tabular}{cccc}
\hline Locus & Linked marker & Primer sequence $\left(5^{\prime} \rightarrow 3^{\prime}\right)$ & Fragment size \\
\hline TaLOX-B1a & LOX16 & F: CCATGACCTGATCCTTCCTT & 489bp \\
& & R: GCGCGGATAGGGGTGGT & \\
TaLOX-B1b & LOX18 & F: ACGATGTGAGTTGTGACTTGTGA & 791bp \\
QLpx.caas-4B & GWM251 & F: CAACTGGTTGCTACACAAGCA & 79bp, 101bp, 113bp, \\
& & R: GGGATGTCTGTTCCATCTTAG & $117 \mathrm{bp}, 125 \mathrm{bp}$ \\
QLpx.caas-1 $A L$ & WMC312 & F: TGTGCCCGCTGGTGCGAAG & $219 \mathrm{bp}, 227 \mathrm{bp}, 235 \mathrm{bp}$, \\
& & R: CCGACGCAGGTGAGCCAAG & $247 \mathrm{bp}$ \\
\hline
\end{tabular}




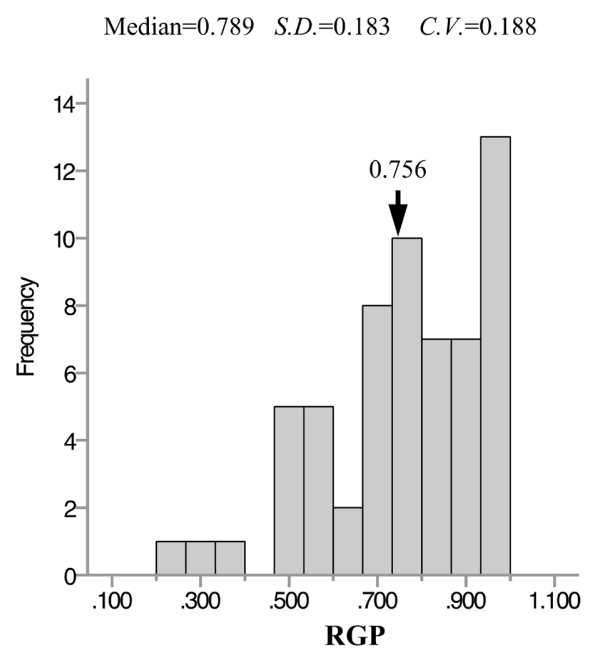

Median $=0.869 \quad$ S.D. $=0.156 \quad$ C.V. $=0.246$

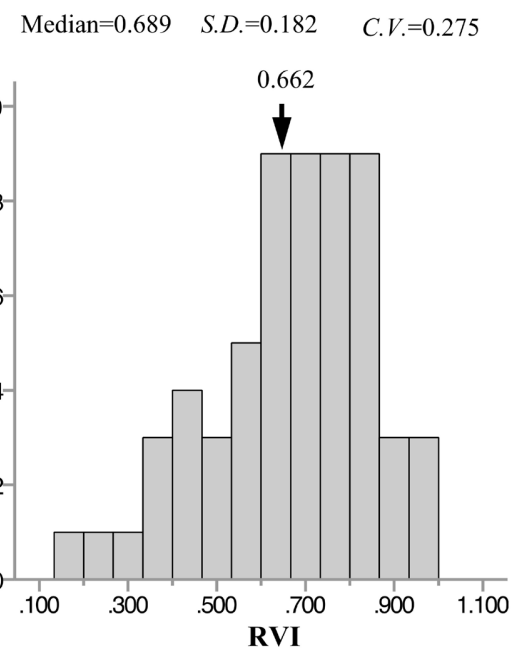

Figure 1. Frequency distribution of seed storability related traits in CIMCOG set. The position pointed by black arrow in X-axis represents phenotypic mean for the population. "Median" means the middle value of the population. "S.D." means its phenotypic standard deviation. "C.V." means coefficient of variation.

Table 2. Correlation analysis between SS-related traits?.

\begin{tabular}{|c|c|c|c|c|c|c|c|c|}
\hline & GP(A) & RGP & $\mathrm{GR}(\mathrm{C})$ & GR(A) & RGR & $\mathrm{VI}(\mathrm{C})$ & VI(A) & RVI \\
\hline $\mathrm{GP}(\mathrm{C})$ & $0.679^{* *}$ & 0.193 & $0.975^{\text {** }}$ & $0.701^{\star *}$ & $0.394^{* *}$ & $0.811^{* *}$ & $0.640^{\star *}$ & $0.370^{* *}$ \\
\hline $\mathrm{GP}(\mathrm{A})$ & & $0.845^{\star *}$ & $0.660^{\star *}$ & $\underline{\underline{0.937^{* *}}}$ & $0.843^{* *}$ & $0.639^{* *}$ & $\underline{\underline{0.928^{* *}}}$ & $0.824^{\star *}$ \\
\hline RGP & & & 0.197 & $0.761^{* *}$ & $\underline{0.865^{* *}}$ & $0.272^{*}$ & $0.771^{\star *}$ & $\underline{0.843^{* *}}$ \\
\hline $\mathrm{GR}(\mathrm{C})$ & & & & $0.719^{* *}$ & $0.313^{*}$ & $0.814^{* *}$ & $0.642^{\star *}$ & $0.387^{\star *}$ \\
\hline GR(A) & & & & & $0.919^{* *}$ & $0.588^{\star *}$ & $\underline{\underline{0.902^{* *}}}$ & $0.867^{* *}$ \\
\hline RGR & & & & & & $0.323^{*}$ & $0.811^{\star \star}$ & $\underline{0.933^{* *}}$ \\
\hline $\mathrm{VI}(\mathrm{C})$ & & & & & & & $0.709^{* *}$ & $0.278^{*}$ \\
\hline $\mathrm{VI}(\mathrm{A})$ & & & & & & & & $0.853^{* *}$ \\
\hline
\end{tabular}

'(A) means AA treatment, (C) means control without AA treatment.

The GP, GR and VI of the seeds of the accessions after AA treatment were significantly correlated with their original GP, GR and VI without AA treatment. In order to reflect the effects caused by AA treatment, the RGP, RGR and RVI was introduced using relative rate between $\mathrm{GA}(\mathrm{A})$ under $\mathrm{AA}$ test and $\mathrm{GA}(\mathrm{C})$ of control, and the correlation coefficient between relative rate and control of GP, GR and VI was decreased by more than at least 2 fold, comparing with the correlation coefficient between relative rate and AA treatment for GP, GR and VI (Table 2: correlation coefficient with dotted underline), while the relationships between relative rate and GP, GR, VI with AA treatment were increased to 0.845 , 0.919 and 0.853 (in Table 2 with bold font). The correlation coefficients among RGP, RGR and RVI were $0.865,0.843$ and 0.933 , respectively (Table 2: correlation coefficient with solid underline). Moreover, the significant positive relationships among GP, GR and VI without AA treatment were also observed with 
high correlation coefficients in CIMCOG set (Table 2: correlation coefficient with wavy underline), and this situation also happened among GP, GR and VI with AA treatment (Table 2: correlation coefficient with double solid underline).

\subsection{Allelic Variation at Lipoxygenase Loci and Phenotypic Effects}

Three loci associated to lipoxygenase activity on chromosomes $1 \mathrm{~A}$ and $4 \mathrm{~B}$ were genotyped using linked or functional markers. The simple sequence repeats (SSR) sites of Xgwm251 and Xwmc312 is tight linked to QLpX.caas-4B and QLPX.caas-1AL related to lipoxygenase activity, respectively. And LOX16 designed from the TaLox-B1 a amplified 489bp PCR fragment in accessions with higher LOX activities, while the marker LOX18 of 791bp PCR fragment was for TaLox-B1b with lower LOX activities. Associations between QLPX.caas- $4 B$, QLPx.caas-1AL, TaLox-B1 and three SS-related traits were executed using the general linear model (GLM). QLpx.caas- $4 B$ was only significantly associated with RVI at the level of $P=0.05$ (Table 3). Both QLPX.caas-1AL and TaLox-B1 were significantly associated with all three SS-related traits at the level of $P=$ 0.001 (Table 3), explaining the percentage of phenotypic variation from $17.5 \%$ (0.175) to $28.3 \%(0.283)$ with GLM (Table 3).

A total of 5 alleles were detected at the locus of $X g w m 215$ with 79, 101, 113, 117 and 125bp PCR fragments in the 60 CIMCOG accessions, the numbers of the 5 allels were 17, 29, 5, 3 and 6, respectively (Table 4). The RGR and RVI of the allele of $A-125 b p$ was significantly higher than those of $A-113 b p$ at $P=0.05$ level (Table 4). At the site of Xwmc312, four alleles $A-219 b p, A-227 b p, A-235 b p$ and $A-247 b p$ were detected with the numbers of 4, 7, 17 and 32 in CIMCOG set, respectively. For RGP, the phenotypic mean of $A-235 b p$ and $A-247 b p$ was significantly higher than that of $A-227 b p$. For RGR, the phenotypic mean of $A-235 b p$ and $A-247 b p$ was significantly higher than that of both $A-219 b p$ and $A-227 b p$. For RVI, the phenotypic mean of $A-247 b p$ was significantly higher than that of both $A-219 b p$ and $A-227 b p$. The phenotypic mean of $A-247 b p$ on all three traits was the highest among the 4 alleles (Table 4). In the CIMCOG set, only 6 accessions carried TaLox-B1a associated to high LOX activity, and the averages of RGP, RGR and RVI of TaLox-B1a were significantly lower than those of TaLoX-B1b, indicating the significant negative relationship between LOX activity and seed storability.

Table 3. Trait-marker associations between three lipoxygenase loci and SS-related traits in CIMCOG set.

\begin{tabular}{ccccccccc}
\hline SS-related trait & Source & Sum of Squares & D.F. & Mean Square & $\mathrm{F}$ value & $P$ value & $-\log (P)$ & $\mathrm{R}^{2}$ \\
\hline RGP & Xgwm251 & 0.239 & 4 & 0.060 & 1.800 & 0.142 & 0.848 & 0.051 \\
& Error & 1.823 & 55 & 0.033 & & & & \\
Total & 2.062 & 59 & & & & & \\
& Xwmc312 & 0.550 & 3 & 0.183 & 6.789 & 0.001 & 3.258 & 0.227 \\
\hline
\end{tabular}




\section{Continued}

\begin{tabular}{|c|c|c|c|c|c|c|c|c|}
\hline & Error & 1.512 & 56 & 0.027 & & & & \\
\hline & Total & 2.062 & 59 & & & & & \\
\hline & $T a L o x B 1$ & 0.460 & 1 & 0.460 & 16.674 & 0.000 & 3.861 & 0.210 \\
\hline & Error & 1.601 & 58 & 0.028 & & & & \\
\hline & Total & 2.062 & 59 & & & & & \\
\hline \multirow[t]{9}{*}{ RGR } & Xgwm251 & 0.224 & 4 & 0.056 & 2.516 & 0.052 & 1.286 & 0.093 \\
\hline & Error & 1.223 & 55 & 0.022 & & & & \\
\hline & Total & 1.956 & 59 & & & & & \\
\hline & Xwmc312 & 0.462 & 3 & 0.154 & 8.770 & 0.000 & 4.134 & 0.283 \\
\hline & Error & 0.984 & 56 & 0.018 & & & & \\
\hline & Total & 1.446 & 59 & & & & & \\
\hline & TaLoxB1 & 0.352 & 1 & 0.352 & 18.632 & 0.000 & 4.203 & 0.230 \\
\hline & Error & 1.095 & 58 & 0.019 & & & & \\
\hline & Total & 1.446 & 59 & & & & & \\
\hline \multirow[t]{9}{*}{ RVI } & Xgwm251 & 0.352 & 4 & 0.088 & 3.014 & 0.026 & 1.593 & 0.120 \\
\hline & Error & 1.604 & 55 & 0.029 & & & & \\
\hline & Total & 1.956 & 59 & & & & & \\
\hline & Xwmc312 & 0.571 & 3 & 0.190 & 7.686 & 0.000 & 3.622 & 0.254 \\
\hline & Error & 1.386 & 56 & 0.025 & & & & \\
\hline & Total & 1.956 & 59 & & & & & \\
\hline & $T a L o x B 1$ & 0.370 & 1 & 0.370 & 13.528 & 0.001 & 3.287 & 0.175 \\
\hline & Error & 1.586 & 58 & 0.027 & & & & \\
\hline & Total & 1.956 & 59 & & & & & \\
\hline
\end{tabular}

Table 4. Multiple comparison of seed storability between alleles at three lipoxygenase loci.

\begin{tabular}{cccccc}
\hline QTL/Marker & Allele & No. & RGP & RGR & RVI \\
\hline Xgwm251 & $A-113 \mathrm{bp}$ & 17 & $0.707 \mathrm{a}$ & $0.764 \mathrm{a}$ & $0.585 \mathrm{a}$ \\
& $A-117 \mathrm{~b} p$ & 29 & $0.753 \mathrm{a}$ & $0.821 \mathrm{ab}$ & $0.651 \mathrm{ab}$ \\
& $A-79 \mathrm{~b} p$ & 5 & $0.806 \mathrm{a}$ & $0.884 \mathrm{ab}$ & $0.701 \mathrm{ab}$ \\
& $A-101 \mathrm{~b} p$ & 3 & $0.728 \mathrm{a}$ & $0.912 \mathrm{ab}$ & $0.745 \mathrm{ab}$ \\
& $A-125 \mathrm{bp}$ & 6 & $0.931 \mathrm{a}$ & $0.966 \mathrm{~b}$ & $0.854 \mathrm{~b}$ \\
& $A-219 \mathrm{~b} p$ & 4 & $0.671 \mathrm{ab}$ & $0.646 \mathrm{a}$ & $0.462 \mathrm{a}$ \\
& $A-227 \mathrm{~b} p$ & 7 & $0.536 \mathrm{a}$ & $0.656 \mathrm{a}$ & $0.502 \mathrm{a}$ \\
& $A-235 \mathrm{bp}$ & 17 & $0.743 \mathrm{~b}$ & $0.827 \mathrm{~b}$ & $0.623 \mathrm{ab}$ \\
& $A-247 \mathrm{bp}$ & 32 & $0.831 \mathrm{~b}$ & $0.890 \mathrm{~b}$ & $0.742 \mathrm{~b}$ \\
& $a$ & 6 & $0.498^{* * *}$ & $0.599^{* * * *}$ & $0.426^{* * *}$ \\
& $b$ & 54 & 0.790 & 0.854 & 0.688 \\
\hline
\end{tabular}

DMRT at $\alpha=0.05$ level. ${ }^{* * *},{ }^{* * * *}$ mean significant difference at $P=0.001$ and 0.0001 levels, respectively. 


\subsection{Haplotypes at Lipoxygenase Loci and Their Phenotypic Effects}

A total of 15 types of allele combination (haplotype) at the loci of QLpx.caas-4B, QLPX.caas- $4 B$ and TaLox-B1 were detected in the CIMCOG set, and 9 out of the 13 haplotypes were distributed in more than 3 accession, as their allele frequencies were not less than $5.0 \%$ (Table 6). The $-[\log (P)]$ of all trait-haplotype associations were more than 4.0 , with the $\mathrm{R}^{2}$ more than $40.0 \%$ in the CIMCOG set, indicating that the three LOX-related loci had additive effects on RGP, RGR and RVI.

The number of accessions carrying each main haplotype varied from 3 to 15 , and 15 accessions out of 60 carried Haplotype-VIII $(117 \mathrm{bp}+247 \mathrm{bp}+\mathrm{b})$, while Haplotype-I, Haplotype-II and Haplotype-III had the lowest haplotype frequencies (Table 5). Significant and large differences were observed among haplotypes on RGP, RGR and RVI for SS (Table 5). For RGP and RGR, the phenotypic mean of Haplotype- $I X$ was significantly higher than the phenotypic mean of the four haplotypes Haplotype-I, -II, -III and -IV. For RVI, the phenotypic mean of Haplotype- $I X$ was significantly higher than that of Haplotype- $I$, - II, - III, - IV, - V and - VI, and there was no significant difference between Haplotype- $I X$ and Haplotype-VII, -VIII (Table 6; Figure 2). Haplotype-IX produced the seeds with the

Table 5. Trait-marker associations between haplotype of lipoxygenase locus and SS-related traits in CIMCOG set.

\begin{tabular}{ccccccccc}
\hline SS-related trait & Source & Sum of Squares & D.F. & Mean Square & F value & $P$ value & $-\log (P)$ & $\mathrm{R}^{2}$ \\
\hline \multirow{2}{*}{ RGP } & Haplotype & 0.806 & 8 & 0.101 & 5.459 & 0.000 & 4.075 & 0.407 \\
& Error & 0.812 & 44 & 0.018 & & & & \\
& Total & 1.617 & 52 & & & & & \\
\multirow{5}{*}{ RGR } & Haplotype & 0.672 & 8 & 0.084 & 7.310 & 0.000 & 5.404 & 0.493 \\
& Error & 0.505 & 44 & 0.011 & & & & \\
& Total & 1.177 & 52 & & & & & \\
\multirow{2}{*}{ RVI } & Haplotype & 0.879 & 8 & 0.110 & 6.280 & 0.000 & 4.685 & 0.448 \\
& Error & 0.769 & 44 & 0.017 & & & & \\
& Total & 1.648 & 52 & & & & &
\end{tabular}

Table 6. Multiple comparison of seed storability between haplotypes at Lox loci.

\begin{tabular}{cccccc}
\hline Haplotype & $X g w m 251+X w m c 312+T a L o x-B 1$ & No. & RGP & RGR & RVI \\
\hline$I$ & $H-113 b p+227 b p+a$ & 3 & $0.450 \mathrm{a}$ & $0.529 \mathrm{a}$ & $0.353 \mathrm{a}$ \\
$I I$ & $H-117 b p+219 b p+b$ & 3 & $0.710 \mathrm{~b}$ & $0.627 \mathrm{a}$ & $0.428 \mathrm{ab}$ \\
$I I I$ & $H-117 b p+227 b p+b$ & 3 & $0.506 \mathrm{a}$ & $0.679 \mathrm{ab}$ & $0.548 \mathrm{bc}$ \\
$I V$ & $H-113 b p+235 b p+b$ & 5 & $0.704 \mathrm{~b}$ & $0.778 \mathrm{bc}$ & $0.561 \mathrm{bc}$ \\
$V$ & $H-117 b p+235 b p+b$ & 7 & $0.772 \mathrm{bc}$ & $0.834 \mathrm{~cd}$ & $0.646 \mathrm{c}$ \\
$V I$ & $H-79 b p+235 b p+b$ & 4 & $0.787 \mathrm{bc}$ & $0.875 \mathrm{~cd}$ & $0.663 \mathrm{c}$ \\
$V I I$ & $H-117 b p+247 b p+b$ & 15 & $0.815 \mathrm{bc}$ & $0.889 \mathrm{~cd}$ & $0.725 \mathrm{~cd}$ \\
$V I I I$ & $H-113 b p+247 b p+b$ & 8 & $0.869 \mathrm{bc}$ & $0.891 \mathrm{~cd}$ & $0.736 \mathrm{~cd}$ \\
$I X$ & $H-125 b p+247 b p+b$ & 5 & $0.942 \mathrm{c}$ & $0.974 \mathrm{~d}$ & $0.878 \mathrm{~d}$ \\
\hline
\end{tabular}

DMRT at $\alpha=0.05$ level. 


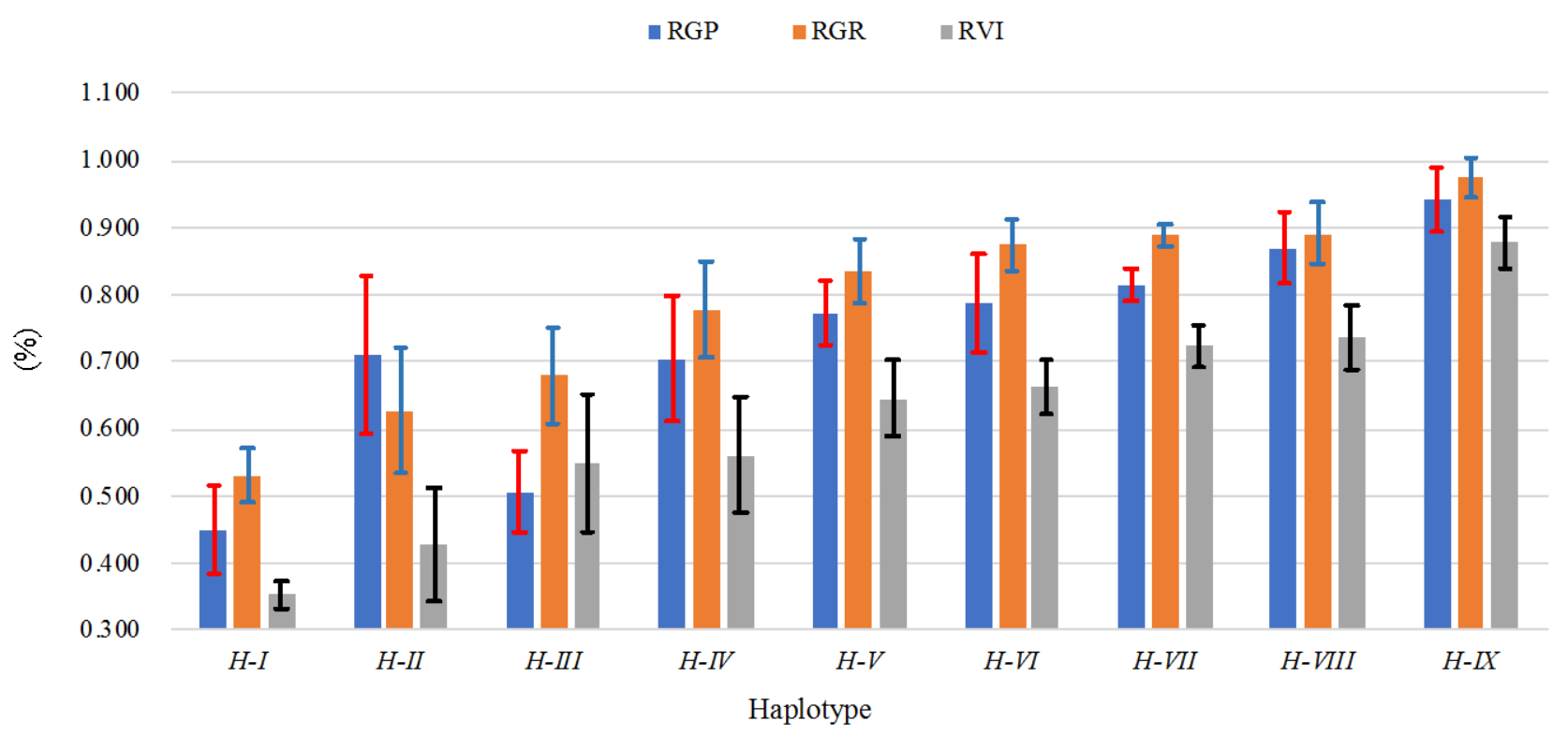

Figure 2. Phenotypic effects of haplotypes at three lipoxygenase loci. Error bars displayed the interval of Mean \pm S.E.

highest RGP, RGR and RVI after AA-treatment (Table 6; Figure 2), suggesting that the allele combination of Xgwm251 $1_{-125 b p}, X w m c 312_{-247 b p}$ and TaLox-B1b was associated with best seed storability. Plants carried Haplotype-I of Xgwm251 $1_{-113 b p}$ $X w m c 312_{-227 b p}$ and TaLox-B1 a generated seed with poorest storability.

\section{Discussion}

The genomic sites $X w m c 312$ of linked to QLPX.caas-1AL, Xgwm251 linked to $Q L p x$.caas- $4 B$ and TaLox- $B 1$ were significantly associated with lipoxygenase activity related genes [23] [24] [25]. With GLM, we also detected significant associations between $X_{W m c 312}$ and TaLox-B1 and all three SS-related traits. For QLpx.caas-4B, the linked SSR marker Xgwm251 was only associated with RVI in the CIMCOG set. However, according to Geng et al. [25], both QLPX.caas-4B and $Q L P x$.caas-1 $A L$ were main QTLs explaining $>25.0 \%$ of the phenotypic variance of LOX activity on average. Geng et al. [23] aligned TaLox-B1 locus to the confidence interval of $Q L p x$.caas- $4 B$ in 2012, indicating that TaLox-B1 might be the candidate gene of $Q L P x$.caas- $4 B$. With the release of high-quality assembly of reference sequence of wheat, the TaLox-B1 was aligned to the physical interval of $30.0 \mathrm{Mb}-30.1 \mathrm{Mb}$ on chromosome 4BS of Chinese Spring (CS) [30], and QLPX.caas-4B was on the interval of Xgwm149 - Xwmc349 [25] with the physical position of $544.6 \mathrm{Mb}-641.0 \mathrm{Mb}$ on chromosome $4 \mathrm{BL}$ of CS [30], while the SSR site Xgwm251 closely linked to the QTL peak of QLPX.caas-4B was at the physical location of $567.7 \mathrm{Mb}$ on $4 \mathrm{BL}$ of Chinese Spring [30], indicating that they were two different genes. And we thought that the only 71 double haploid lines used for QTL mapping and the lack of high-quality Ref-Seq assembly of CS caused the mis-alignment of QLPX.caas- $4 B$.

Geng et al. [25] analyzed the association between LOX activity and QLPX.caas- 
$1 A L, Q L p x . c a a s-4 B$ using their linked markers $X w m c 312, X g w m 251$ in 198 Chinese wheat cultivars and lines. The allele of $A-247 \mathrm{bp}$ at the site of $X g w m 312$ had significant lower LOX activity than its $A-235 b p$, and the allele of $A-247 b p$ belonged to the group with lowest LOX activity. At the site of $\mathrm{Xgwm} 251$, the allele of $A-125 b p$ had lower LOX activity than $A-117 b p$, and lines carrying $A-125 b p$ have the lowest average LOX activity. And the mean LOX activity of the haplotype $X g w m 251_{-125 b p}+X w m c 312_{-247 b p}$ belonged to the DMRT-testing group with lowest LOX activity. The TaLox- $B 1 b$ had significant lower mean LOX activity than TaLox-B1a [23]. Lipoxygenase activity is often negatively related to seed longevity/storability [13] [14] [15] [17] [31] [32]. Hence, these alleles at the three loci with lowest LOX activity in Geng et al. [23] [25], such as Xgwm251 ${ }_{-125 b p}$, $X$ wmc312 $2_{-247 b p}$ and TaLox-B1b, had the highest RGP, RGR and RVI with the best seed storability in our study, and the wheat accessions carrying the haplotype of $X g w m 251_{-125 b p}+X w m c 312_{-247 b p}+$ TaLox-B1 b produced seeds with the best storability, comparing with other haplotypes. However, no significant associations between RGP/RGR and Xgwm251 were detected by GLM procedure in the panel of CIMMYT core wheat germplasm, mostly due to the small population size of the panel [33] and the existence of other quantitative trait loci except for Lox genes [12] [34].

Good seed storability is beneficial to preserving seeds for germplasm banks and providing reliable crop seeds to farmers. Among 5 accessions carrying Hap-

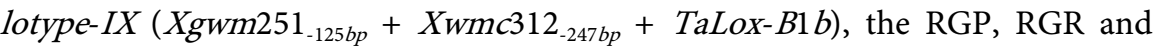
RVI of accessions No. 14, 31, 35 and 57 were more than $0.95,0.95$, and 0.83 , and the seed coat color of them were white [11]. These accessions with the lowest LOX activity could cut the greater loss of carotenoids than vitamin E during breadmaking that induced by high LOX activity [18], which could also be used for flour end-quality improvement.

\section{Conclusion}

Among three reported LOX activity related QTLs/genes, QLPX.caas-1AL and $T a L O X-B 1$ were also significantly associated with three SS-related parameters in CIMMYT core wheat germplasm panel (CIMCOG) with only 60 accessions. A total of 9 haplotypes were detected at three lipoxygenase activity related gene lo$\mathrm{ci}$, and we found that the haplotype of XgWm251-125bp$+X w m c 312_{-247 b p}+T a-$ $L o x-B 1 b$ produced seeds with the best storability in the CIMCOG.

\section{Acknowledgements}

This study was supported by Sichuan Province Science and Technology Department (2020YJ0469 \& 2017JY0077), the Sichuan Province Science and Technology Department Crops Breeding Project (2020YFSY0049 \& 2021YFYZ0002), the Project of Resource Exploitation and Breeding Scab Resistant Wheat by Sichuan Provincial Finance Department, and Talent fund (2019LJRC007) and Frontier-science Fund (2019QYXK034) of Sichuan Academy of Agricultural 
Sciences.

\section{Conflicts of Interest}

The authors declare no conflicts of interest regarding the publication of this paper.

\section{References}

[1] Liu, Y., Bino, R.J., van der Burg, W.J., Groot, S.P.C. and Hilhorst, H.W.M. (1996) Effects of Osmotic Priming on Dormancy and Storability of Tomato (Lycopersicon esculentum Mill.) Seeds. Seed Science Research, 6, 49-55.

https://doi.org/10.1017/S0960258500003020

[2] Harrington, J.F. (1972) Seed Storage and Longevity. In: Kozlowski, T.T., Ed., Seed Biology, Insects, and Seed Collection, Storage, Testing and Certification 3, Academic Press, New York, 145-245. https://doi.org/10.1016/B978-0-12-395605-7.50009-0

[3] Copeland, L.C. and McDonald, M.B. (2001) Principles of Seed Science and Technology. 4th Edition, Kluwer Academic Publishers, Dordrecht.

https://doi.org/10.1007/978-1-4615-1619-4

[4] Clerkx, E.J.M., El-Lithy, M.E., Vierling, E., Ruys, G.J., Blankestijn-De Vries, H., Groot, S.P.C., Vreugdenhil, D. and Koornneef, M. (2004) Analysis of Natural Allelic Variation of Arabidopsis Seed Germination and Seed Longevity Traits between the Accessions Landsberg erecta and Shakdara, Using a New Recombinant Inbred Line Population. Plant Physiology, 135, 432-444. https://doi.org/10.1104/pp.103.036814

[5] Zeng, D.L., Guo, L.B., Xu, Y.B., Yasukumi, K., Zhu, L.H. and Qian, Q. (2006) QTL Analysis of Seed Storability in Rice. Plant Breeding, 125, 57-60. https://doi.org/10.1111/j.1439-0523.2006.01169.x

[6] Xue, Y., Zhang, S.Q., Yao, Q.H., Peng, R.H., Xiong, A.S., Li, X., Zhu, W.M., Zhu, Y.Y. and Zha, D.S. (2008) Identification of Quantitative Trait Loci for Seed Storability in Rice (Oryza sativa L.). Euphytica, 164, 739-744. https://doi.org/10.1007/s10681-008-9696-3

[7] Li, L., Lin, Q., Liu, S., Liu, X., Wang, W., Hang, N.T., Liu, F., Zhao, Z., Jiang, L. and Wan, J. (2012) Identification of Quantitative Trait Loci for Seed Storability in Rice (Oryza sativa L.). Plant Breeding, 131, 739-743. https://doi.org/10.1111/j.1439-0523.2012.02007.x

[8] Dargahi, H., Tanya, P. and Srinives, P. (2014) Mapping of the Genomic Regions Controlling Seed Storability in Soybean (Glycine max L.). Journal of Genetics, 93, 365-370. https://doi.org/10.1007/s12041-014-0381-0

[9] Nagel, M., Rosenhauer, M., Willner, E., Snowdon, R.J., Friedt, W. and Börner, A. (2011) Seed Longevity in Oilseed Rape (Brassica napus L.) - Genetic Variation and QTL Mapping. Plant Genetic Resources. Characterization and Utilization, 9, 260-263. https://doi.org/10.1017/S1479262111000372

[10] Wu, S.Y. and Zhou, G.Y. (2011) Comparison of Aging Characteristics and Longevity of Wheat Seeds in Vacuum and Non-Vacuum Packages Storage in Mid-Term Low-Temperature Genebank. Acta Agronomica Sinica, 37, 1109-1115. https://doi.org/10.3724/SP.J.1006.2011.01109

[11] Zhang, J., Xiang, S. and Wan, H. (2021) Negative Association between Seed Dormancy and Seed Longevity in Bread Wheat. American Journal of Plant Sciences, 12, 347-365. https://doi.org/10.4236/ajps.2021.123022 
[12] Rehman Arif, M.A., Nagel, M., Neumann, K., Kobiljski, B., Lohwasser, U. and Börner, A. (2012) Genetic Studies of Seed Longevity in Hexaploid Wheat Using Segregation and Association Mapping Approaches. Euphytica, 186, 1-13. https://doi.org/10.1007/s10681-011-0471-5

[13] Wilson, D.O. and McDonald, M.B. (1986) The Lipid Peroxidation Model of Seed Aging. Seed Science and Technology, 14, 269-300.

[14] Li, J., Zhang, Y., Yu, Z., Wang, Y., Yang, Y., Liu, Z., Jiang, J., Song, M. and Wu, Y. (2007) Superior Storage Stability in Low Lipoxygenase Maize Varieties. Journal of Stored Products Research, 43, 530-534. https://doi.org/10.1016/j.jspr.2006.09.005

[15] Zhang, Y., Yu, Z., Lu, Y., Wang, Y., She, D., Song, M. and Wu, Y. (2007) Effect of the Absence of Lipoxygenase Isoenzymes on the Storage Characteristics of Rice Grains. Journal of Stored Products Research, 43, 87-91. https://doi.org/10.1016/j.jspr.2005.11.004

[16] Dong, Z., Feng, B., Liang, H., Rong, C., Zhang, K., Cao, X., Qin, H., Liu, X., Wang, T. and Wang, D. (2015) Grain-Specific Reduction in Lipoxygenase Activity Improves Flour Color Quality and Seed Longevity in Common Wheat. Molecular Breeding, 35, 150. https://doi.org/10.1007/s11032-015-0347-9

[17] Leenhardt, F., Lyan, B., Rock, E., Boussard, A., Potus, J., Chanliaud, E. and Remesy, C. (2006) Genetic Variability of Carotenoid Concentration, and Lipoxygenase and Peroxidase Activities among Cultivated Wheat Species and Bread Wheat Varieties. European Journal of Agronomy, 25, 170-176. https://doi.org/10.1016/j.eja.2006.04.010

[18] Leenhardt, F., Lyan, B., Rock, E., Boussard, A., Potus, J., Chanliaud, E. and Remesy, C. (2006) Wheat Lipoxygenase Activity Induces Greater Loss of Carotenoids than Vitamin E during Breadmaking. Journal of Agricultural and Food Chemistry, 54, 1710-1715. https://doi.org/10.1021/jf052243m

[19] Trufanov, V.A., Permyakova, M.D., Pshenichnikova, T.A., Ermakova, M.F., Davydov, V.A., Permyakov, A.V. and Berezovskaya, E.V. (2007) The Effect of Inter-Cultivar Substitution of Wheat Triticum aestivum L. Chromosomes on Lipoxygenase Activity and Its Correlation with the Technological Properties of Flour. Applied Biochemistry and Microbiology, 43, 91-97.

https://doi.org/10.1134/S0003683807010164

[20] Krinsky, N.I. (1989) Antioxidant Functions of Carotenoids. Free Radical Biology and Medicine, 7, 617-635. https://doi.org/10.1016/0891-5849(89)90143-3

[21] Hart, G.E. and Langston, P.J. (1977) Chromosomal Location and Evolution of Isozyme Structural Genes in Hexaploid Wheat. Heredity, 39, 263-277. https://doi.org/10.1038/hdy.1977.67

[22] Hessler, T.G., Thomson, M.J., Benscher, D., Nachit, M.M. and Sorrells, M.E. (2002) Association of a Lipoxygenase Locus, $L p x-B 1$, with Variation in Lipoxygenase Activity in Durum Wheat Seeds. Crop Science, 42, 1695-1700. https://doi.org/10.2135/cropsci2002.1695

[23] Geng, H.W., Xia, X.C., Zhang, L.P., Qu, Y.Y. and He, Z.H. (2012) Development of Functional Markers for a Lipoxygenase Gene TaLOX-B1 on Chromosome 4BS in Common Wheat. Crop Science, 52, 568-576. https://doi.org/10.2135/cropsci2011.07.0365

[24] Carrera, A., Echenique, V., Zhang, W., Helguera, M., Manthey, F., Schrager, A., Picca, A., Cervigni, G. and Dubcovsky, J. (2007) A Deletion at the $L p x$ - B1 Locus Is Associated with Low Lipoxygenase Activity and Improves Pasta Color in Durum 
Wheat (Triticum turgidum ssp. durum). Journal of Cereal Science, 45, 67-77. https://doi.org/10.1016/j.jcs.2006.07.001

[25] Geng, H., Zhang, Y., He, Z., Zhang, L., Appels, R., Qu, Y. and Xia, X. (2011) Molecular Markers for Tracking Variation in Lipoxygenase Activity in Wheat Breeding. Molecular Breeding, 28, 117-126. https://doi.org/10.1007/s11032-010-9466-5

[26] ISTA (2008) International Rules for Seed Testing. International Seed Testing Association, Bassersdorf.

[27] Abdul-Baki, A.A. and Anderson, J.D. (1973) Vigor Determination in Soybean Seed by Multiple Criteria. Crop Science, 13, 630-633. https://doi.org/10.2135/cropsci1973.0011183X001300060013x

[28] Qu, J., Li, J., Zhang, Z., Zheng, J., Li, S., Peng, Z., Yang, W. and Wan, H. (2018) Allele-Specific PCR Markers for Distinguishing High Molecular Weight Glutenin Subunit $D^{t} x 5$ of Aegilops tauschii from $D x 5$ of Common Wheat. Molecular Plant Breeding, 9, 44-52

[29] Wan, H., Wen, W., Li, J. and Yang, W. (2014) Optimization of AS-PCR Identification System for HMW-GS $D^{t} x 1.5$ of Synthetic Wheat Developed with Durum Wheat and Aegilops tauschii and Its Genetic Analysis. Molecular Plant Breeding, 12, 629-637. (In Chinese)

[30] Iwgsc, A.R., Eversole, K., et al. (2018) Shifting the Limits in Wheat Research and Breeding Using a Fully Annotated Reference Genome. Science, 61, eaar7191.

[31] Huang, J., Cai, M., Long, Q., Liu, L., Lin, Q., Jiang, L., Chen, S. and Wan, J. (2014) OsLOX2, a Rice Type I Lipoxygenase, Confers Opposite Effects on Seed Germination and Longevity. Transgenic Research, 23, 643-655. https://doi.org/10.1007/s11248-014-9803-2

[32] Li, Z., Gao, Y., Lin, C., Pan, R., Ma, W., Zheng, Y., Guan, Y. and Hu, J. (2018) Suppression of LOX Activity Enhanced Seed Vigour and Longevity of Tobacco (Nicotiana tabacum L.) Seeds during Storage. Conservation Physiology, 6, coy047. https://doi.org/10.1093/conphys/coy047

[33] Oraguzie, N.C., Rikkerink, E.H.A., Gardiner, S. and de Silva, N. (2007) Association Mapping in Plants. Springer, Berlin. https://doi.org/10.1007/978-0-387-36011-9

[34] Pshenichnikova, T.A., Osipova, S.V., Permyakova, M.D., Mitrofanova, T.N., Trufanov, V.A., Lohwasser, U., Röder, M. and Börner, A. (2008) Mapping of Quantitative Trait Loci (QTL) Associated with Activity of Disulfide Reductase and Lipoxygenase in Grain of Bread Wheat Triticum aestivum L. Russian Journal of Genetics, 44, 567-574. https://doi.org/10.1134/S1022795408050098 